\title{
COMMENTARY
}

\section{NEW TRICKS FOR AN OLD WORKHORSE by Bernard Dixon}

$\mathrm{L}$ actobacillus plantarum is a versatile beast, touching life at many points. In the laboratory, it has been used as an exquisitely sensitive test organism for assaying pantothenic acid, nicotinic acid, biotin, and other vitamins. In the world of the gastronome, it plays a major part in the fermenting of pickles and sauerkraut, and also crops up in the starter cultures used to produce Grana and Swiss cheeses. On the farm, it is a key member of the microbial consortium employed to make silage. It seems to occupy significant niches in waste treatment communities toosometimes in symbiotic association with Streptococcus faecalis, which receives folic acid from the lactobacillus in exchange for the phenylalanine it requires. Cow pats and the human mouth are two of the many other diverse habitats from which $L$. plantarum can usually be isolated.

Not surprisingly, Bergey's Manual of Determinative Bacteriology records at least eight aliases for this agreeably versatile microbe. In many less reliable tomes, it has been libelled for its alleged inability to cope with hydrogen peroxide in the environment. In fact, as Fred Skinner and Louis Quesnel point out in their book Streptococci (Academic Press), L. plantarum given proper nourishment is more than capable of surviving exposure to $\mathrm{H}_{2} \mathrm{O}_{2}$. Quite a character.

As with other lactobacilli, however, relatively little is yet known about the basic genetics of $L$. plantarum. This is a pity, because it and its peers are tempting targets for genetic manipulation, through which the organisms could be programmed to express useful heterologous proteins. If engineered strains of $L$. plantarum were able to secrete such proteins into the growth medium, a particular temptation would be to incorporate genes coding for enzymes permitting the organisms to grow on much cheaper substrates than those required by the slightly fastidious $L$. plantarum in its native state. Although the wild type is unable to degrade starch, for example, strains capable of thriving on inexpensive starchy feedstocks might be of considerable commercial value.

These challenges have now been taken up. In the last two or three years, researchers at several centers have begun to develop techniques for manipulating lactobacilli genetically. Reports of the expression of genes coding for antibiotic resistance and plasmid replication have appeared in the literature accordingly. For my money, one of the most promising avenues of work is at Cranfield Institute of Technology in the U.K., where lactobacilli are being coaxed into expressing metabolic enzymes, too.

The first step was, of course, to achieve a high frequency of transformation, and this has now been accomplished by means of electroporation in $L$. plantarum. Now two Cranfield researchers, Phil Warner assisted by Sian Jones, have embarked on a program of work to incorporate genes encoding the production of amylases, with the aim of extending the substrate range of the organism to include polysaccharides. Their first choice was $\alpha$-amylase, because it can be assayed easily, and because it has been used as a model protein in studies on both the mechanisms of protein secretion and the relationship between the structure and stability of proteins.

The Cranfield duo's initial efforts to introduce the $\alpha$ amylase gene of Bacillus amyloliquefaciens into L. plantarum were less than fully successful. Using plasmid pGK12 as the cloning vector, they found recombinants carrying the gene, but these were extremely unstable. When Warner and Jones turned to plasmid pIL253, however, they picked up a recombinant, pCIT602, that could be subcultured repeatedly on starch-containing medium with no loss of the plasmid. Unlike pGK12, pIL253 does not replicate via single-stranded DNA. Originally designed for gene cloning in Lactococcus, it can clearly be used just as efficiently in $L$. plantarum and does not become unstable when foreign DNA is inserted.

Warner and Jones obtained the B. amyloliquefaciens $\alpha$ amylase gene as a cloned fragment in plasmid pSB6 isolated from its $B$. subtilis host. They then ligated it to plasmid pIL253, yielding plasmid pCIT602, which they used to transform protoplasts of a plasmid-free $B$. subtilis lacking $\alpha$-amylase activity. Transformants were selected with erythromycin and screened for production of the enzyme. Agarose gel electrophoresis of the plasmid DNA revealed the presence of a recombinant plasmid, pCIT602, of the expected size. Finally, the duo used electroporation to introduce the plasmid into $L$. plantarum at a relatively high frequency. Transformants, selected with erythromycin and linocomycin, contain the plasmid.

Although the genetically manipulated organism grew on MRSB medium supplemented with starch, and although the Cranfield group certainly found $\alpha$-amylase in the culture supernatants, the level of expression was significantly lower than that observed in $B$. subtilis. It is even possible that lysis or leakage from the organisms, rather than genuine secretion, may be responsible for the appearance of enzyme activity. Nevertheless, Phil Warner believes that the engineered $L$. plantarum could be a prototype for the development of lactobacilli able to use an increased range of substrates in commercial fermentations. He and his colleagues are now sequencing the promoter and signal regions of the $\alpha$-amylase gene, and hope to improve expression in $L$. plantarum by optimising the promoter sequences.

Although Warner and Jones's project on pCIT602 was supported in the U.K. by the Agricultural and Food Research Council, the U.S. company Ag-Bag is now helping to finance Cranfield's work with lactobacilli. AgBag is particularly interested in tactics to improve the quality of silage and to prevent its spoilage. Perhaps overwintering cattle, rather than human gourmets or fermentation technologists, will be the first to benefit from genetically engineered alterations in the lifestyle of Lactobacillus plantarum and its close relatives. 